\title{
Macrophage-derived netrin-1 drives adrenergic nerve-associated lung fibrosis
}

\author{
Ruijuan Gao, ${ }^{1,2}$ Xueyan Peng, ${ }^{1}$ Carrighan Perry, ${ }^{1}$ Huanxing Sun, ${ }^{1}$ Aglaia Ntokou, ${ }^{3}$ Changwan Ryu, ${ }^{1}$ Jose L. Gomez, \\ Benjamin C. Reeves, ${ }^{1}$ Anjali Walia, ${ }^{1}$ Naftali Kaminski, ${ }^{1}$ Nir Neumark, ${ }^{1}$ Genta Ishikawa, ${ }^{1}$ Katharine E. Black, ${ }^{4}$ Lida P. Hariri, ${ }^{4,5}$ \\ Meagan W. Moore, ${ }^{1}$ Mridu Gulati, ${ }^{1}$ Robert J. Homer ${ }^{1,6}$ Daniel M. Greif, ${ }^{3,7}$ Holger K. Eltzschig, ${ }^{8}$ and Erica L. Herzog ${ }^{1,6}$ \\ 'Section of Pulmonary, Critical Care, and Sleep Medicine, Department of Internal Medicine, Yale School of Medicine, New Haven, Connecticut, USA. 'Department of Oncology, Institute of Medicinal \\ Biotechnology, Chinese Academy of Medical Sciences \& Peking Union Medical College, Beijing, China. ${ }^{3}$ Section of Cardiology, Department of Internal Medicine, Yale School of Medicine, New Haven, \\ Connecticut, USA. " Division of Pulmonary and Critical Care Medicine, and ${ }^{5}$ Department of Pathology, Massachusetts Ceneral Hospital, Harvard Medical School, Boston, Massachusetts, USA. ${ }^{6}$ Department \\ of Pathology, and 'Department of Genetics, Yale School of Medicine, New Haven, Connecticut, USA. ${ }^{8}$ Department of Anesthesiology, University of Texas at Houston Medical School, Houston, Texas, USA.
}

\begin{abstract}
Fibrosis is a macrophage-driven process of uncontrolled extracellular matrix accumulation. Neuronal guidance proteins such as netrin-1 promote inflammatory scarring. We found that macrophage-derived netrin-1 stimulates fibrosis through its neuronal guidance functions. In mice, fibrosis due to inhaled bleomycin engendered netrin-1-expressing macrophages and fibroblasts, remodeled adrenergic nerves, and augmented noradrenaline. Cell-specific knockout mice showed that collagen accumulation, fibrotic histology, and nerve-associated endpoints required netrin-1 of macrophage but not fibroblast origin. Adrenergic denervation; haploinsufficiency of netrin-1's receptor, deleted in colorectal carcinoma; and therapeutic $\boldsymbol{\alpha}_{1}$ adrenoreceptor antagonism improved collagen content and histology. An idiopathic pulmonary fibrosis (IPF) lung microarray data set showed increased netrin-1 expression. IPF lung tissues were enriched for netrin-1+ macrophages and noradrenaline. A longitudinal IPF cohort showed improved survival in patients prescribed $\alpha_{1}$ adrenoreceptor blockade. This work showed that macrophages stimulate lung fibrosis via netrin-1-driven adrenergic processes and introduced $\alpha_{1}$ blockers as a potentially new fibrotic therapy.
\end{abstract}

\section{Introduction}

Fibrotic pathology is common to nearly all organ systems and contributes to approximately $45 \%$ of deaths worldwide $(1,2)$. Its development involves prolonged or recurrent epithelial injury that activates fibroblasts through poorly understood mechanisms. This process is to varying degrees orchestrated by macrophages (3), a myeloid population implicated in the scarring of diverse organs such as the lung (4), skin (5), liver (6), and kidney (7). Macrophages are equally important in the central nervous system, where microglia support the formation, maintenance, and pruning of axons and synapses (8) via an array of functions that may be similarly tuned in peripheral nerves (9). Although nerve-derived signals have been recently shown to regulate the phenotype of macrophages in an array of organs (10-14), corresponding input from macrophages to lung innervation remains undescribed.

A substantial hindrance to curing fibrosis is our limited understanding of the mechanisms through which injury and sterile inflammation produce a scar. One realm of interest in this area is neuronal guidance proteins (NGPs), a diverse and conserved family of molecules, including netrins, semaphorins, ephrins, and neuropilins, which together with their receptors are increasingly

Conflict of interest: ELH consults for Boehringer Ingelheim, Genentech, Merck; and has grant funding from Boehringer Ingelheim, Sanofi, Biogen Idec, and Bristol Myers. Copyright: (c) 2021, American Society for Clinical Investigation.

Submitted: January 23, 2020; Accepted: September 10, 2020; Published: January 4, 2021 Reference information: J Clin Invest. 2021;131(1):e136542.

https://doi.org/10.1172/JCl136542. studied at the interface of injury and repair (15). Unlike the unidirectional interactions induced by cytokines and chemokines, the bidirectional responses of NGPs occur via attractive or repulsive receptors, which enact inflammatory remodeling in the lung (16), liver (17), adipose tissue (18), and kidney (19). In addition to these roles, NGPs were originally described as patterning growth cone movement at developing axon tips $(20,21)$ and at least one NGP, the laminin-like protein netrin-1 (NTN1), mediates peripheral nerve repair via the attractive receptor, deleted in colorectal cancer (DCC) $(22,23)$. These properties are recapitulated in postnatal development, where the interaction between NTN1 and DCC controls adrenergic innervation of maturing vessels and viscera (24). Interestingly, although adrenergic nerve-derived signals influence immune activation in several disease models $(12,25,26)$, a reciprocal role for immune cells in controlling this type of innervation in peripheral organs remains obscure.

In this study, we proposed that macrophage-derived NTN1 contributes to fibrosis through its axonal guidance and remodeling functions. In mice, fibrosis due to inhaled bleomycin engendered NTN1-expressing macrophages, remodeled adrenergic nerves, and augmented noradrenaline. In cell-specific knockout mice, collagen accumulation, fibrotic histology, and nerve-associated endpoints required NTN1 of macrophage but not fibroblast origin. Adrenergic denervation, haploinsufficiency of the NTN1 receptor DCC, and therapeutic $\alpha_{1}$ adrenoreceptor antagonism improved collagen content and histology. An existing microarray data set from patients with idiopathic pulmonary fibrosis (IPF) showed increased NTN1 expression. IPF lung tissues were enriched for 
$\mathrm{NTN}^{+}$macrophages and noradrenaline. In a longitudinal IPF cohort, patients prescribed $\alpha_{1}$ adrenergic antagonists experienced improved survival. These findings showed that $\mathrm{NTN} 1^{+}$drives adrenergic nerve-associated fibrosis in organs such as the lung and introduces $\alpha_{1}$ adrenoreceptor antagonism as a potentially new treatment for IPF and related disorders.

\section{Results}

NTN1-expressing macrophages accumulate in the fibrotic lung. NGPs such as NTN1 participate in the remodeling of multiple tissues, but the source and mechanism of this function is not fully known. To address this knowledge gap, we used the fibrotic lung as a modeling system and randomized wild-type (WT) mice to receive a single inhaled dose of vehicle control or $1.25 \mathrm{U} / \mathrm{kg}$ of pharmacological grade bleomycin. After 14 days, immunohistochemistry of vehicle- and bleomycin-challenged lungs revealed $\mathrm{NTN}^{+}$cells with the morphology of macrophages, fibroblasts, epithelia, and endothelia that were more numerous in the fibrotic lung (Figure 1, A and B; and Supplemental Figure 1; supplemental material available online with this article; https://doi.org/10.1172/ JCI136542DS1). When these observations were quantified using FACS, enumeration of macrophages $\left(\mathrm{F} 4 / 80^{+} \mathrm{CD} 11 \mathrm{~b}^{+} \mathrm{CD} 11 \mathrm{c}^{+}\right)$, fibroblasts $\left(\mathrm{CD}^{-} 5^{-} \mathrm{ColI} \alpha^{+}\right)$, epithelia $\left(\mathrm{CD} 45^{-} \mathrm{CD} 326^{+} \mathrm{CD} 31^{-}\right)$, and endothelia $\left(\mathrm{CD}^{-} 5^{-} \mathrm{CD} 31^{+} \mathrm{CD} 326^{-}\right)$revealed a large increase in the proportion of $\mathrm{NTN}^{+}$macrophages, with a much smaller increase seen in endothelial cells and no change in either fibroblasts or epithelial cells (Figure 1, C-F). Total quantities were also enhanced, with $\mathrm{NTN}^{+}$macrophages and fibroblasts increasing dramatically and the NTN1 ${ }^{+}$epithelium and endothelium expanding to a much smaller extent (Figure 1, C-F). Because both percentages and quantities of $\mathrm{NTN}^{+}$macrophages were enhanced after bleomycin, we presumed these cells to be a critical part of the fibrotic response and took the additional step of confirming their presence with imaging mass cytometry (IMC). IMC is a powerful method pairing heavy metal-tagged antibodies with inductively coupled mass spectroscopy and time-of-flight mass cytometry to allow unbiased, quantitative histology unencumbered by artifacts such as autofluorescence (27). Using this approach, NTN1 ${ }^{+}$macrophages were also found to accumulate after bleomycin exposure (Supplemental Figure 2), a further indication that these cells may drive fibrosis in adult organs such as the lung.

NTN1 derived from macrophages, but not fibroblasts, regulates experimentally induced lung fibrosis. Because NTN1-expressing macrophages were most substantially increased after bleomycin, we surmised that they may drive pulmonary fibrosis. To determine the validity of this hypothesis, we generated several strains of mice with cell-specific Ntn1 deficiency and subjected them to the bleomycin model. To delete $N t n 1$ from macrophages, $N t n 1^{f / f l}$ mice were crossed with LysM-Cre animals to delete Ntn1 in cells with an active lysozyme 2 (Lyz2) promoter, which targets myeloid cells and a small population of type II pneumocytes (28). Specific deletion of NTN1 in macrophages was confirmed by Western blotting (Supplemental Figure 3). Baseline lung morphology and macrophage content were unchanged between unchallenged animals with a WT Ntn1 locus (LysM-Cre $\mathrm{NtnI}^{+/+}$, unless stated otherwise, from here on called "intact") or deleted (LysM-Cre Ntn1 $1^{f / f l}$, from here on called "deleted," Supplemental Figure 4). After bleomycin, LysM-Cre Ntn1 $1^{t / f l}$ lungs contained significantly reduced collagen compared with LysM-Cre $\mathrm{NtnI}^{+/+}$ lungs (Figure $1 \mathrm{G}$ and Supplemental Figure 5). Improvements in histological endpoints such as modified Ashcroft scores (MAS, Figure $1 \mathrm{H}$ ) and trichrome staining (Figure 1, I and J) were also observed. To determine the specificity of this contribution, we also generated a model of pan-fibroblast Ntn1 deletion, produced by crossing $N t n 1^{f / / f l}$ mice to ColIa2-CreER mice (ColIa2-CreER Ntn1 fl/ft), which failed to affect these same fibrotic endpoints. Specifically, relative to identical animals receiving corn oil control ("intact"), ColI 2 -CreER Ntn1 $1^{f / / f l}$ mice receiving 7 days of tamoxifen ("deleted") before bleomycin displayed equivalent collagen accumulation (Figure 1K), modified Ashcroft scores (Figure 1L), and fibrotic histology (Figure 1, M and $\mathrm{N}$ ) despite a significant reduction in $\mathrm{NTN}^{+}$fibroblasts (Supplemental Figure 6). These observations indicate that for maximal induction of fibrosis in this model, NTN1 derived from myeloid cells such as macrophages is required and NTN1 derived from collagen I $\alpha 2-$ producing cells such as fibroblasts is dispensable.

Macrophage-derived NTN1 participates in adrenergic nerve remodeling. NTN1 regulates processes related to inflammatory fibrosis in peripheral organs, but a contribution of its most well-described function, neuronal guidance, has not been proposed. Because NTN1 controls adrenergic innervation of visceral organs and the remodeling of injured peripheral nerves, we evaluated these endpoints in the bleomycin model. In naive and bleomycin-challenged WT mouse lungs, confocal imaging of $150 \mu \mathrm{m}$ thick sections revealed adrenergic nerves identified by the rate-limiting enzyme in catecholamine synthesis, tyrosine hydroxylase (TH, Figure 2, A-D and Supplemental Figure 7), and the pan-neuronal marker, protein-gene-product 9.5 (PGP9.5, Figure 2, E-G). These structures were juxtaposed to $\alpha$-smooth muscle actin-positive $\left(\alpha \mathrm{SMA}^{+}\right)$ airways and vessels, as well as within the lumens of larger airways, in the distribution that has been previously reported for this class of neurons $(13,14)$ (Figure 2, A and B and Supplemental Figure 8). In the fibrotic lung, they were accompanied by aberrantly located $\mathrm{TH}^{+}$linear structures clustered in parenchymal regions (Figure 2, C, $\mathrm{D}$, and $\mathrm{H}$ ). The possibility of these filaments being remodeled yet functional adrenergic nerves was supported by detection of the neurotransmitter noradrenaline, whose presence in the lung derives mainly from local nerves or adrenal sources, being increased in lung tissue out of proportion to adrenaline, which is only delivered to the lungs from a systemic source (Figure 2I). No such change in the ratio of noradrenaline to adrenaline was observed in bleomycin-challenged bronchoalveolar lavage (BAL) fluid (Supplemental Figure 9). All of these findings were absent in the lungs of myeloid Ntn1deleted mice after bleomycin (Figure 2, J-O and Supplemental Figure 10). Although cultured Ntn1-deficient macrophages displayed the expected alterations in cytokine production $(29,30)$ (Supplemental Figure 11), the in vivo findings did not appear to stem from baseline differences in noradrenaline/adrenaline or in alterations in the magnitude of injury, acute inflammation, or accumulation of fibrosis-promoting macrophages expressing the mannose receptor C-type 1 (MRC1) (31) at early, intermediate, or late time points, respectively (Supplemental Figure 12). When viewed in combination, these data suggest that NTN1's role in fibrosis may involve a previously unrecognized mechanism involving adrenergic nerves.

Adrenergic denervation attenuates experimentally induced lung fibrosis. We next reasoned that if nerve-derived noradrenaline is 
A

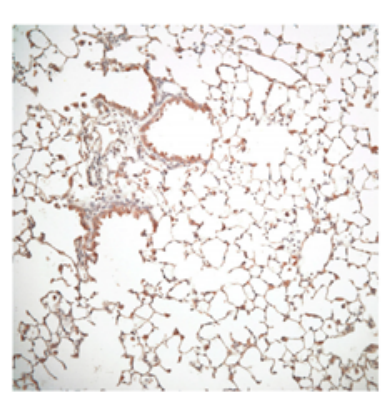

Fibroblasts

D

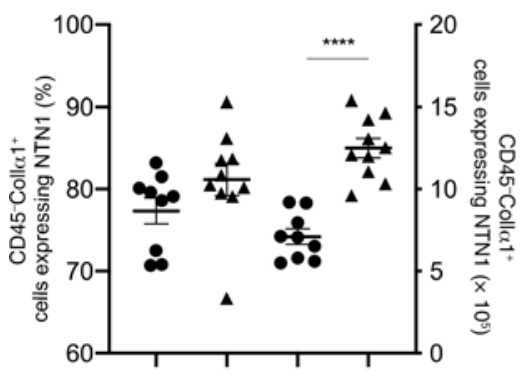

B

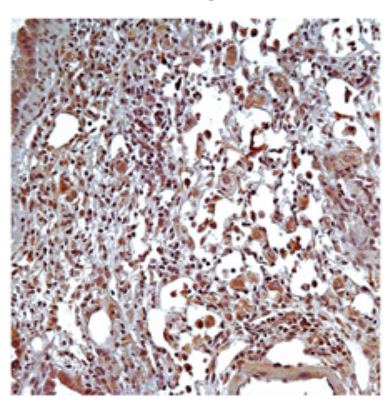

Epithelia

E

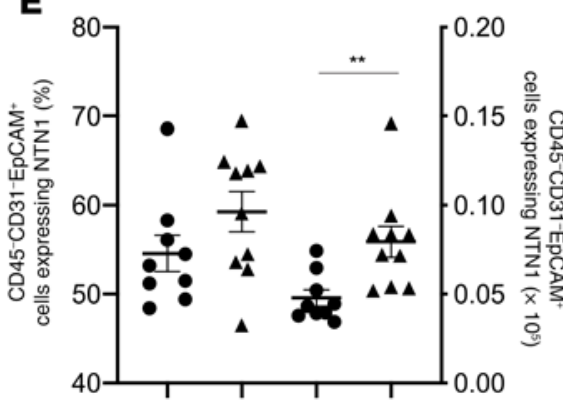

C Macrophages

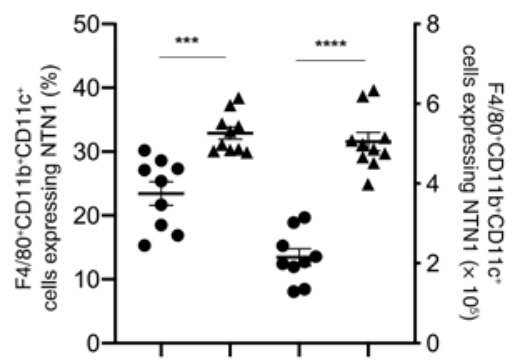

Endothelia

$\mathbf{F}$

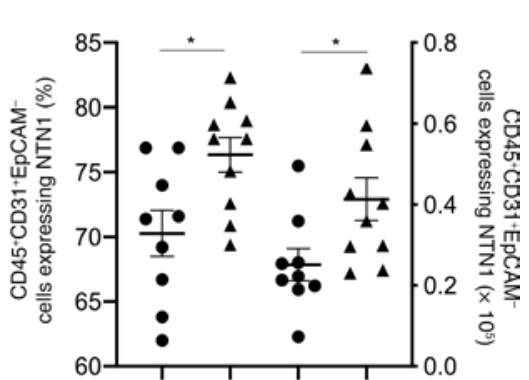

Bleomycin

Macrophage Ntn1

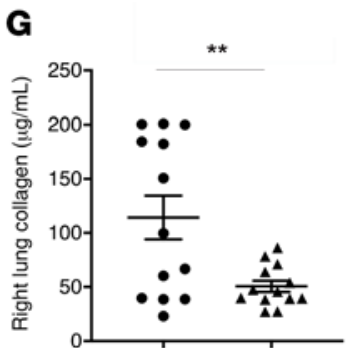

H
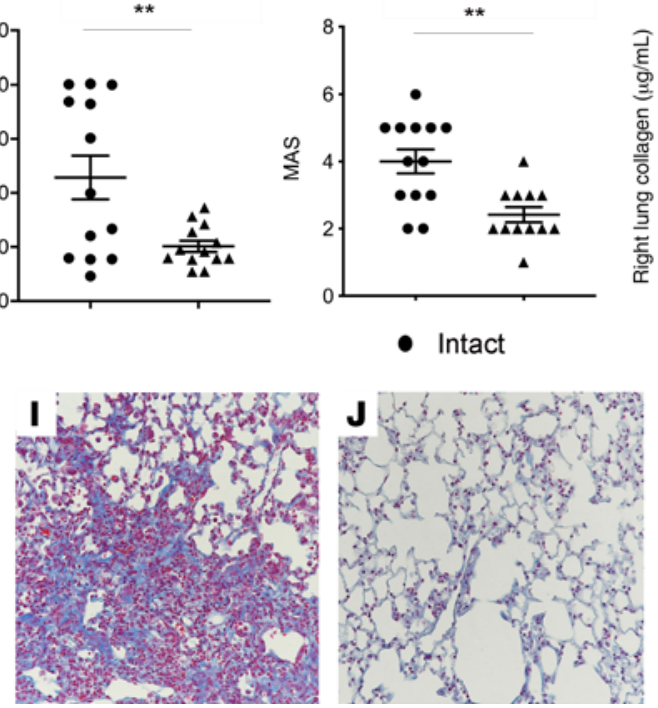

K

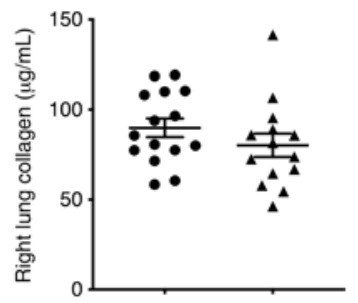

\ Deleted

Fibroblast Ntn1

L
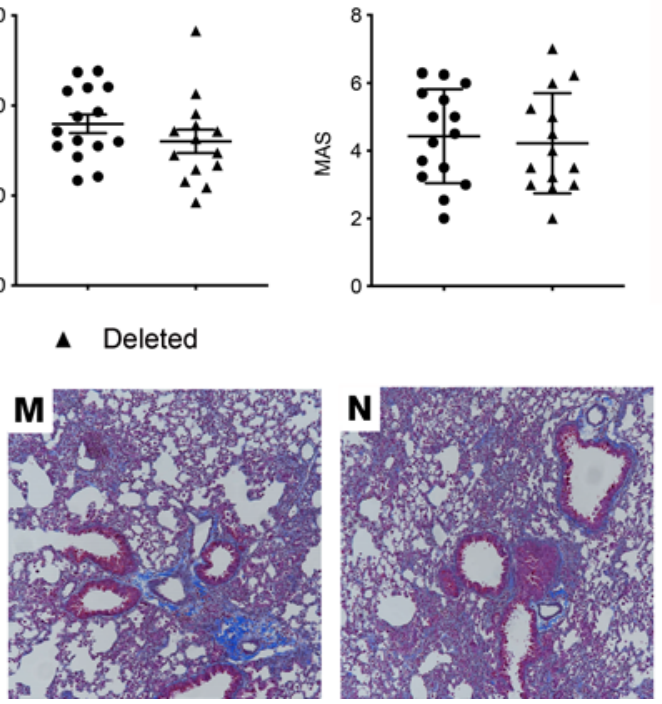

Figure 1. Macrophage-derived NTN1 in experimentally induced lung fibrosis. (A and B) WT mice received a single orotracheal dose of vehicle or $1.25 \mathrm{U} / \mathrm{kg}$ pharmacological grade bleomycin and were followed for 14 days. After the mice were euthanized, the lungs underwent immunohistochemical detection of NTN1 (brown stain). Vehicle- (A) and bleomycin-challenged lungs (B) contained multiple populations of NTN1+ cells that appeared more numerous in the bleomycin condition. Slides were counterstained with hematoxylin and are shown as $\times 10$ original magnification. (C-F) Flow cytometric detection of NTN1+ macrophages (C, identified with the markers $\mathrm{F} 4 / 80^{+} \mathrm{CD} 11 \mathrm{~b}^{+} \mathrm{CD} 11 \mathrm{c}^{+}$), fibroblasts ( $\mathbf{D}$, identified with the markers $\mathrm{CD} 45^{-} \mathrm{Coll}^{-} 1^{+}$), epithelial cells (E, identified with the markers $\mathrm{CD} 45^{-}$ $\left.\mathrm{CD}^{-} 1^{-} \mathrm{EpCAM}\right)$ and endothelial cells (F, identified with the markers CD45-EpCAM-CD31+) in single-cell suspensions prepared from lungs of WT mice challenged with vehicle (round symbol) or bleomycin (triangle). Left axis shows percentages, and right axis shows quantities. (G-J) Relative to mice with "intact" expression

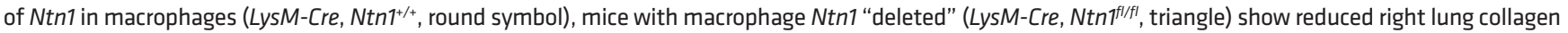
(G, $P=0.0056$, 2-tailed Student's $t$ test) and, in a separate group of mice, both MAS (H, $P=0.0013$ by 2-tailed Student's $t$ test) and trichrome staining (I, "intact" and J, "deleted"). (K-N) Relative to mice with "intact" expression of Ntn1 in fibroblasts (corn oil-treated Colla2-CreER, Ntn fl/ff animals, round symbol), mice with Ntn1 "deleted" in fibroblasts (tamoxifen-treated Colla2-CreER, Ntn $7^{f / f l}$ mice, triangle) show unchanged right lung collagen (K), and in a separate group of mice, both MAS (L) and trichrome staining ( $\mathbf{M}$, "intact" and $\mathbf{N}$, "deleted"). ${ }^{*} P<0.05,{ }^{*} P<0.01,{ }^{* *} P<0.001$, and ${ }^{* * * *} P<0.0001$. Data are shown as mean \pm SEM. 


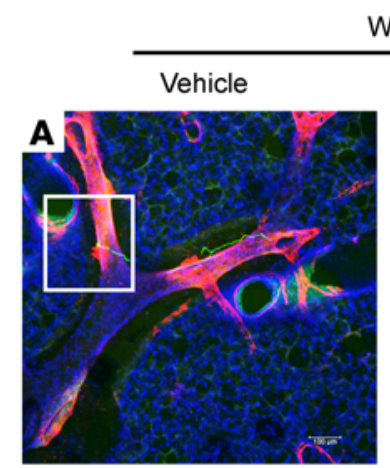

WT
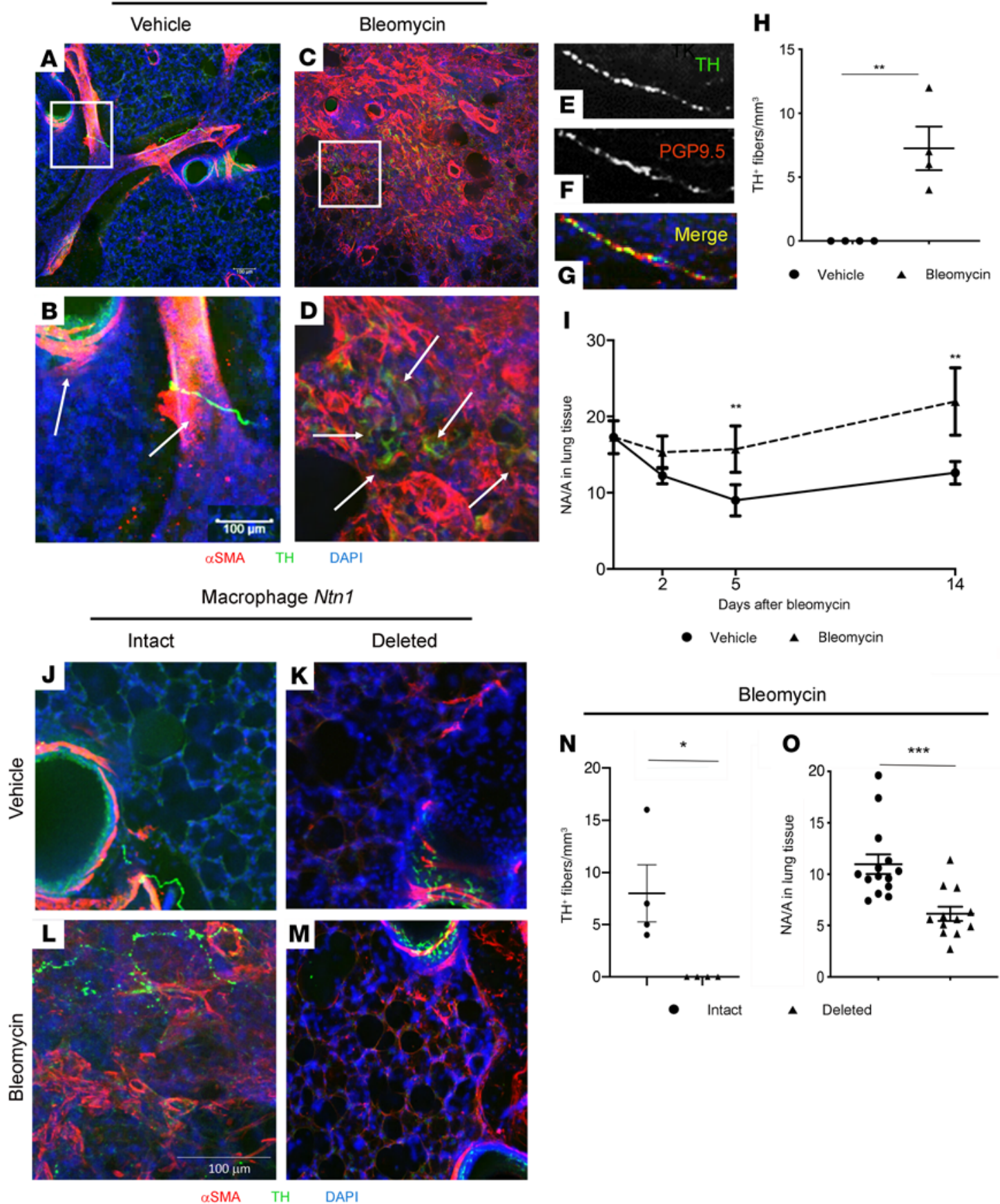

Figure 2. Adrenergic nerve remodeling in experimentally induced lung fibrosis. (A-D) Immunofluorescence detection of $\alpha$ SMA (red), TH (green), and DAPI (blue) in vehicle- ( $\mathbf{A}$ and $\mathbf{B}$ ) and bleomycin-challenged (C and $\mathbf{D}$ ) lungs from WT mice at the 14-day time point. In the vehicle-exposed sample in $\mathbf{B}$, white arrows indicate $\mathrm{TH}$ staining in an airway (left) and artery (right). In the bleomycin image in $\mathbf{D}$, white arrows indicate $\mathrm{TH}^{+}$linear structures in the parenchyma that are not associated with airways or vessels. (E-G) Colocalization of TH (E, green) with the pan-neuronal marker PGP9.5 (F, red). Merge shows overlay with DAPI staining (G, blue). Original magnification, $\times 60$. (H) Comparison of $\mathrm{TH}^{+}$fibers in the lung parenchyma of WT mice challenged with vehicle (round symbol, not easily visible because of fibers being undetectable) and bleomycin (triangle, $P=0.0053$, 2-tailed Student's $t$ test). (I) Comparison of the ratio between noradrenaline and adrenaline (ratio is NA/A) in WT mice challenged with vehicle (round symbol) and bleomycin (triangle) revealed that relative to vehicle-treated groups, the NA/A ratio in lung tissue was increased at the 5 -day $(P<0.01)$ and 14 -day $(P<0.01)$ time points (2-way ANOVA with Tukey's post hoc test). (J-M) Immunofluorescence detection of $\alpha$ SMA (red), TH (green), and DAPI (blue) in the lungs of vehicle-challenged mice with macrophage Ntn1 intact (J) or deleted $(\mathbf{K})$, and bleomycin-challenged mice with macrophage Ntn1 intact (L) or deleted (M). (N and $\mathbf{O}$ ) Relative to mice with intact Ntn1, the lungs of macrophagedeleted Ntn1 mice displayed reduced detection of parenchymal TH fibers $(\mathbf{F}, P=0.032$, 2-tailed Student's $t$ test) and NA/A ratio (G, $P=0.0005,2$-tailed Student's $t$ test). Scale bars: $100 \mu \mathrm{m}$ in all images. For $\mathbf{H}$ and $\mathbf{I}$ and $\mathbf{N}$ and $\mathbf{O},{ }^{*} P<0.05,{ }^{*} P<0.01$, and ${ }^{* * *} P<0.001$. Data are shown as mean \pm SEM. 
profibrotic, then adrenergic denervation should block fibrosis. To test this hypothesis, we compared mice treated with either the sympathetic neurotoxin 6-hydroxydopamine hydrochloride (6-OHDA) or vehicle control (L-ascorbic acid) (32) for a 2-week period. Denervated lungs displayed close to absent $\mathrm{TH}^{+}$nerve content around airways and blood vessels (Figure 3, A-D), along with reduced lung tissue noradrenaline/adrenaline (Figure $3 \mathrm{E}$ ), but sustained content of macrophages expressing either NTN1 or MRC1 (Supplemental Figure 13). When mice that did or did not receive 6-OHDA were evaluated in the bleomycin model after 14 days, substantially reduced collagen content was found in the denervated lungs (Figure 3F). Although we cannot rule out a direct effect of 6-OHDA treatment on immune activation and fibroproliferation, because the denervated lungs were so markedly protected, it is both possible and likely that a process linked to adrenergic nerves, perhaps related to macrophage-derived NTN1, participates in pulmonary fibrosis.

Dcc haploinsufficiency attenuates adrenergic nerve remodeling and pulmonary fibrosis. To evaluate a direct effect of NTN1 on adrenergic nerve biology, we investigated its receptor DCC. This transmembrane dependence receptor is implicated in adrenergic nerve development (24) and sensory nerve repair (23), but has not been evaluated in fibrotic disease. In support of a potential role in pulmonary fibrosis, Dcc expression was found to be significantly increased in whole lung lysates prepared from bleomycin-challenged lungs, where it colocalized with $\mathrm{TH}$ in airway lumens after bleomycin administration (Supplemental Figure 14). Because this observation suggested a potential link to nerve remodeling and fibrosis, we employed a spontaneous mouse model of Dcc haploinsufficiency $\left(D c c^{\text {kanga/+ }}\right)$. These animals show reduced expression of $D c c$ without the severe developmental deformities seen in $D c c^{\text {kanga/kanga }}$ animals (33), and are therefore suitable for studies in adult mice. Baseline lung noradrenaline/adrenaline (Supplemental Figure 15) and adrenergic nerve content (Supplemental Figure 15) were equivalent between $D c c^{+/+}$and $D c c^{\text {kanga/+ }}$ mice. However, after bleomycin, $D c c^{\text {kanga/t }}$ lungs contained reductions in the noradrenaline/adrenaline ratio (Figure $3 \mathrm{G}$ ) and in ectopically located $\mathrm{TH}^{+}$structures (Figure 3, H-J). Furthermore, fibrotic indices such as collagen content (Figure 3K), MAS (Figure 3L), and trichrome staining (Figure 3, M and N) were also ameliorated. These data provide evidence that macrophage-derived NTN1 impacts adrenergic nerve remodeling and its association with lung fibrosis through a receptor-mediated interaction with DCC.

Therapeutic $\alpha_{1}$ adrenoreceptor antagonism suppresses lung fibrosis. Because a critical deficiency in the field of fibrosis is the availability of efficacious, well-tolerated, and inexpensive medications, we aimed to illuminate potentially new therapeutic approaches. In considering this goal, we thought that targeting the NTN1-DCC interaction - while mechanistically sound - would be difficult because of the lack of an existing therapeutic pipeline. Sympathectomy is similarly infeasible because of the anatomic challenge of interrupting the sympathetic trunk when it enters the lung and the obvious concern for off-target effects with chemical denervation. As a result, the most attractive, rational, and direct application of our work is the repurposing of safe and well-tolerated adrenoreceptor antagonists that are already approved by the FDA and available in generic form. Based on noradrenaline's $\alpha_{1}$ adrenergic receptor binding properties (34), as well as previous work demonstrating a fibrostimulatory function for this receptor class in otherwise normal rat lungs (35), we tested $\alpha_{1}$ adrenergic receptor antagonism (also called " $\alpha_{1}$ blockers") in the bleomycin model. FACS-based enumeration of the $3 \alpha_{1}$ adrenergic receptor subtypes (ADRA1A, ADRA1B, and ADRA1D) was first performed. Because expression of ADRA subtypes on leukocyte function is implicated in a number of inflammatory conditions $(36,37)$, we interrogated these receptors on $\mathrm{CD}_{4} 5^{+}$cells after bleomycin (Figure $4, \mathrm{~A}-\mathrm{F}$ ). Here we found only negligible expression of ADRA1A in both conditions (Figure 4, A and $\mathrm{B}$ ), with an increase in quantities but not percentages of CD $45^{+}$ cells expressing ADRA1B during fibrosis (Figure 4, C and D). In contrast, we found a substantial increase in both the quantities and percentages of $\mathrm{CD}_{4} 5^{+} \mathrm{ADRA} 1 \mathrm{D}^{+}$cells after bleomycin (Figure 4, E and $\mathrm{F}$ ). In addition, $\mathrm{CD} 45^{-} \mathrm{ColI} \alpha 1^{+}$fibroblasts expressed all 3 receptors at baseline and in a manner that was increased after bleomycin (Supplemental Figure 16, A-C). Epithelial cells possessed close to undetectable or absent expression of ADRA1 subtypes (data not shown). Endothelial cells showed minimal expression of ADRA1A (data not shown) and an increase in quantities of endothelial cells that expressed ADRA1D but not ADRA1B after bleomycin (Supplemental Figure 16, D and E). In an attempt to target these receptors, bleomycin-challenged mice were then randomized to receive therapeutic dosing of the $\alpha_{1}$ adrenoreceptor antagonist terazosin, which targets all ADRA1 subtypes (38), or vehicle control. In a regimen of daily dosing initiated at day 5 and continued until the mice were euthanized at day 14 , terazosin significantly reduced collagen accumulation (Figure 4G and Supplemental Figure 17A), MAS (Figure $4 \mathrm{H}$ ), and trichrome staining (Figure 4 , I and J). Similar to currently available FDA-approved antifibrotics (39), when terazosin was started at the 10-day time point and continued to 21 days, no effect was seen, indicating a mechanism of action involving active fibrogenesis rather than reversal of existing disease (Supplemental Figure 17B). These data show that the fibrosis associated with NTN1-driven, adrenergic nerve-derived noradrenaline responds to treatment with $\alpha_{1}$ adrenergic blockade.

Increased NTN1 gene expression in IPF lungs. Our work's clinical relevance was then probed in a publicly available microarray data set generated from samples collected under the Lung Genomics Research Consortium (LGRC) protocol (40). Data were available for 119 individuals with IPF and 50 controls. Demographic and physiological characteristics are presented in Table 1. Examination of patient variables revealed the groups to be matched for gender, but the IPF cohort was significantly older with evidence of physiological impairment based on the percentage of predicted forced vital capacity (FVC\%) and diffusion capacity of the lung for carbon monoxide (DLCO\%). Individuals with IPF had higher NTN1 expression than controls that persisted despite adjustment for age and lung function (Figure 5A). These findings were confirmed in archived lung biopsy samples obtained from the Yale Lung Repository (TYLR), where relative to the unaffected margins of nodule resections from patients that were found to have a nonmalignant process, biopsies obtained from patients with IPF contained increased expression of NTN1 relative to the internal control $\beta$-actin gene, ACTB (Figure $5 \mathrm{~B}$ ).

Increased NTN1 protein expression in IPF macrophages. To assess the source of this increase in NTN1, we performed NTN1 

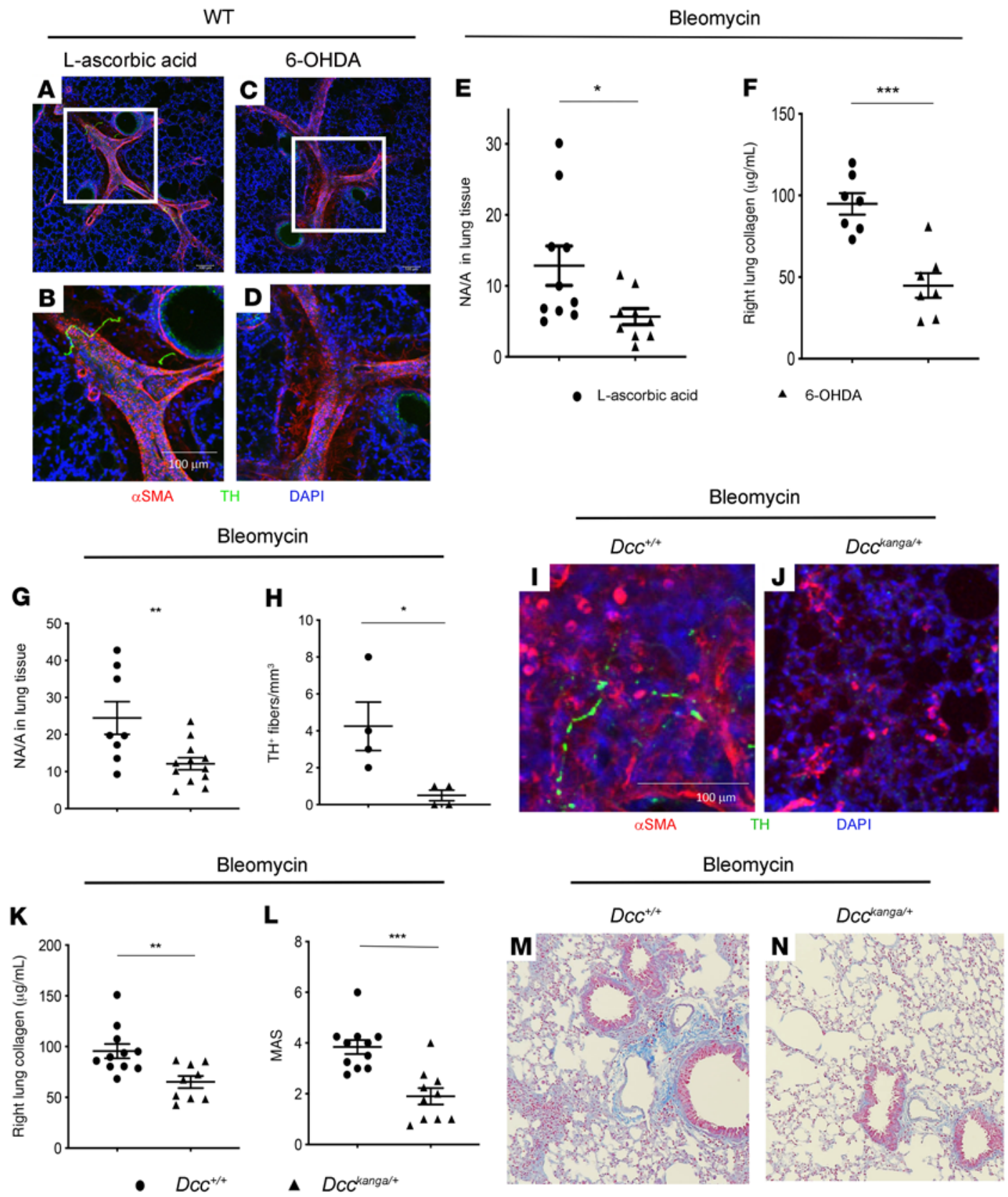

Figure 3. Contributions of adrenergic nerves and Dcc to experimentally induced lung fibrosis. (A-D) Immunofluorescence detection of $\alpha$ SMA (red), TH (green), and DAPI (blue) in the lungs of unchallenged mice treated with either L-ascorbic acid (A and B) or 6-OHDA (C and $\mathbf{D})$. In each low-power image, the boxed region is enlarged in the panel underneath. Scale bars: $100 \mu \mathrm{m}$. (E and F) Compared with bleomycin-exposed mice treated with L-ascorbic acid only (vehicle, round symbol), mice treated with 6-OHDA (triangle) contained reduced lung tissue NA/A (E, $P=0.0349,2$-tailed Student's $t$ test) and right lung collagen (F, $P=0.0003$, 2-tailed Student's $t$ test). (C) Relative to bleomycin-challenged mice with intact expression of Dcc (round symbol), mice that were heterozygote for the spontaneous Dcc kanga/+ mutation (triangle) showed reduced lung tissue NA/A ( $P=0.0073$, 2-tailed Student's $t$ test). (H) Compared with $D c c^{+/+}$mice $(n=4)$, parenchymal detection of $\mathrm{TH}^{+}$fibers was reduced in $D c c^{\text {kanga/+ }}$ mice $(P=0.032$, 2-tailed Student's $t$ test). (I and J) Immunofluorescence detection of $\alpha \mathrm{SMA}$ (red), TH (green), and DAPI (blue) in the lungs of bleomycin-exposed $D c c^{+/+}(\mathbf{I})$ and $D c c^{\text {kanga/+ }}(\mathbf{J})$ mice. Scale bars: $100 \mu \mathrm{m}$. (K-N) After bleomycin, compared with the lungs of $D c c^{+/+}$ mice, Dcc kanga/+ mouse lungs contained less collagen (K, $P=0.0046$, 2-tailed Student's $t$ test), reduced MAS (L, $P=0.0002$, 2-tailed Student's $t$ test), and ameliorated trichrome staining ( $\mathbf{M}$ and $\mathbf{N}$ ). For all graphs, ${ }^{*} P<0.05$, ${ }^{* *} P<0.01$, and ${ }^{* *} P<0.001$. Data are shown as mean \pm SEM.

immunofluorescence on archived lung biopsy tissues from TYLR. Here, NTN1 was found to colocalize with the macrophage marker Mac1 in control (Figure 5, C and D) and fibrotic samples (Figure 5, $\mathrm{E}$ and $\mathrm{F}$ ), with a significant increase in the number of $\mathrm{NTN} 1^{+} \mathrm{Mac1}^{+}$ cells seen in the IPF subjects (Figure 5G). These findings linked the IPF disease state with macrophage-derived NTN1.
IPF lungs are enriched for noradrenaline. Next, we measured noradrenaline and adrenaline in tissues derived from lungs that were unsuitable for use as transplant donors (control) or obtained from IPF patients at the time of transplant. As with the animal studies described above, the noradrenaline/adrenaline ratio was substantially increased in IPF lungs (Figure $5 \mathrm{H}$ ), 

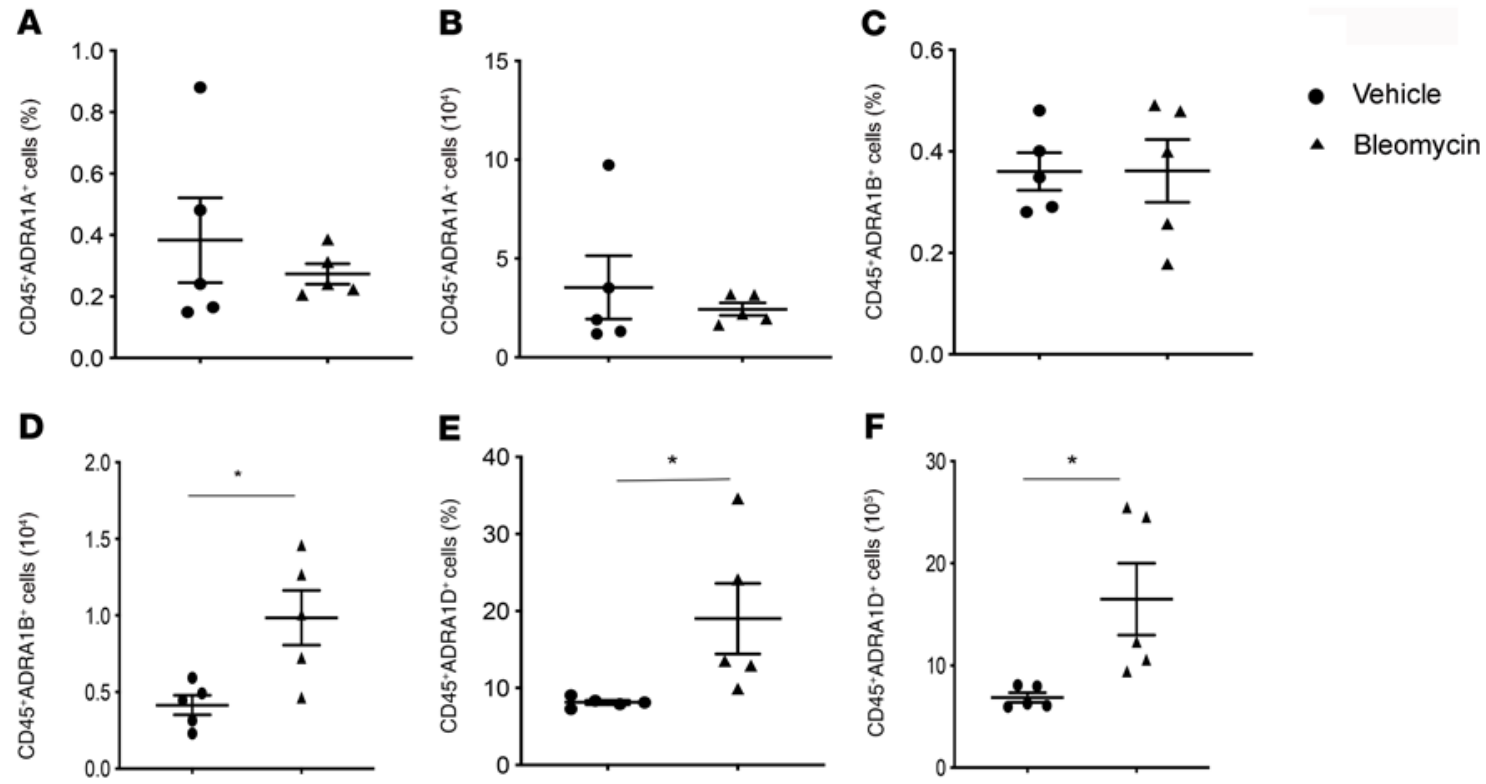

Bleomycin
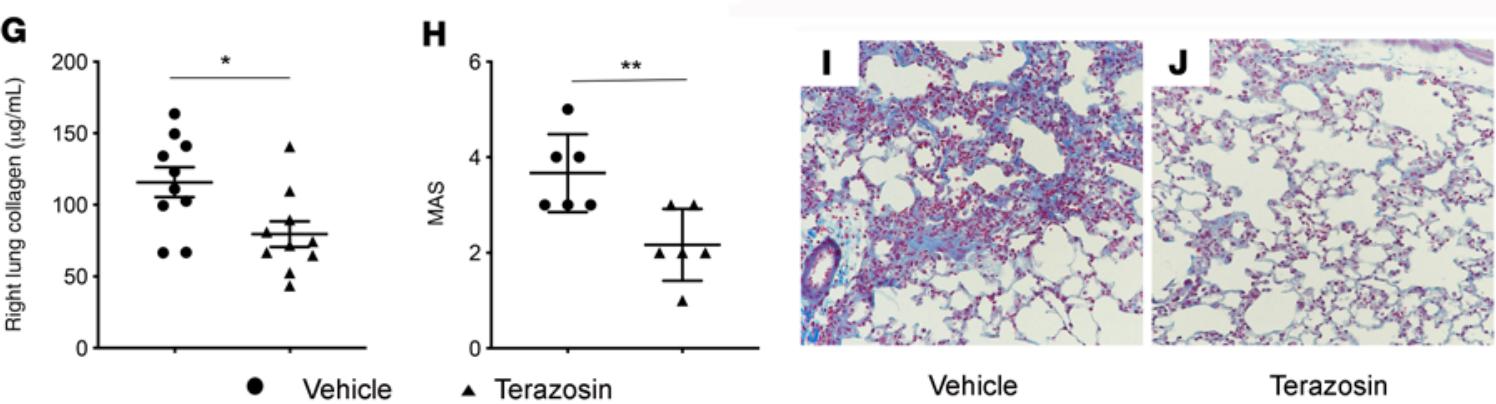

Figure 4. Therapeutic benefit of $\alpha_{1}$ adrenoreceptor antagonism in experimentally induced lung fibrosis. (A-F) The lungs of WT mice that were sacrificed 14 days after a single inhaled dose of vehicle control (round symbol) or $1.25 \mathrm{U} / \mathrm{kg}$ bleomycin (triangular symbol) underwent FACS-based enumeration of percentages (A) and quantities (B) of CD45+ hematopoietic cells expressing ADRA1A; (C and $\mathbf{D})$ percentages (C) and quantities (D) of CD45+ hematopoietic cells expressing ADRA1B; and (E and $\mathbf{F})$ percentages $(\mathbf{E})$ and quantities $(\mathbf{F})$ of $C D 45^{+}$hematopoietic cells expressing ADRA1D. Only percentages of ADRA1D-expressing cells were significantly increased 14 days after inhaled bleomycin (E, $P=0.0454$, 2-tailed Student's $t$ test), whereas an increase was seen in quantities of both $C D 45^{+} A D R-$ $\mathrm{A}^{1} \mathrm{~B}^{+}\left(\mathbf{D}, P=0.0172\right.$, 2-tailed Student's $t$ test) and $\mathrm{CD}_{4} 5^{+}$ADRA1D+ cells ( $\mathbf{F}, P=0.0267$, 2-tailed Student's $t$ test). (G-J) Relative to bleomycin-exposed mice treated with vehicle control (round symbol), bleomycin-challenged mice treated with daily i.p. injections of $1 \mathrm{mg} / \mathrm{kg}$ terazosin (triangle) showed improvements in right lung collagen (G, $P=0.0165$, 2-tailed Student's $t$ test) and in separate experiments, MAS (H, $P=0.0079$, 2-tailed Student's $t$ test) and trichrome staining (I and J). In all comparisons, ${ }^{*} P<0.05$ and ${ }^{* *} P<0.01$. Data are shown as mean \pm SEM. Original magnification of images, $\times 10$.

suggesting a relationship between NTN1, macrophages, and noradrenaline in this setting.

Use of $a_{1}$ adrenoreceptor antagonists is associated with improved survival in IPF. Finally, we confirmed the therapeutic potential of $\alpha_{1}$ adrenoreceptor antagonism in IPF by examining the historical data of IPF subjects followed at the Yale Interstitial Lung Disease Center of Excellence. At the time of analysis, 264 subjects with IPF had been enrolled in our registry. To avoid confounders such as evolving clinical practice and immunosuppression use before 2011, our analysis focused on newly referred, treatment-naive subjects enrolled under the 2011 European Respiratory Society/American Thoracic Society criteria (41). Medication review identified no patients using an $\alpha_{1}$ adrenoreceptor antagonist for a cardiovascular indication. However, a substantial number of patients were prescribed these drugs for a prostate-related indication. Because these individuals were male $(n=22)$, further analysis was restricted to this gender to result in the moderately sized cohort of 89 patients described in Supplemental Table 1. This entirely male cohort was mostly white (93\%) with a mildly impaired FVC\%; severely impaired predicted DLCO\%; and a gender, age, and physiology (GAP) index score of 4, which is associated with a $42 \%$ 3-year mortality (42).

All-cause mortality was then assessed during a 1000-day longitudinal follow-up, which is within the median survival length for this cohort, in patients who were $(n=22)$ or were not taking ( $n=67) \alpha_{1}$ antagonists (Table 2). Of the 22 patients using $\alpha_{1}$ blockers, 17 were prescribed tamsulosin and 5 were prescribed terazosin. Notably, patients taking $\alpha_{1}$ adrenoreceptor antagonists trended toward being older, yet exhibited significantly better FVC\%, DLCO\%, and GAP scores (Table 2). Receiver operator curve (ROC) analysis revealed $\alpha_{1}$ antagonist use to reliably dichotomize subjects for all-cause mortality (Supplemental 
Table 1. Demographic characteristics of the LGRC cohort

\begin{tabular}{|c|c|c|c|}
\hline & IPF & Control & $P$ value \\
\hline$n$ & 119 & 50 & \\
\hline Age median [IQR] & $64[58-68]$ & $47[33-60]$ & $<0.01$ \\
\hline Male sex $\%(n)$ & $65 \%(77)$ & $54 \%(27)$ & 0.26 \\
\hline FVC\% predicted & $62[50-72]$ & $\mathrm{n} / \mathrm{a}$ & \\
\hline DLCO\% predicted & $44[29-59]$ & $\mathrm{n} / \mathrm{a}$ & \\
\hline NTN1 ( $\log _{2}$ of normalized array) & $6.3[6.2-6.5]$ & $6.1[5.9-6.2]$ & $1.4 \times 10^{-9}$ \\
\hline
\end{tabular}

Data are presented as median and IQR. FVC\% = percentage of predicted forced vital capacity. DLCO\% = percentage of predicted diffusion capacity of the lung for carbon monoxide.

Figure 18A). Kaplan-Meier analysis showed that $\alpha_{1}$ antagonism conferred a significant survival benefit (Supplemental Figure 18B) that persisted after covariate adjustments for age, FVC\%, DLCO \%, and GAP index (Figure 5I). These data impart the clinical impact of our basic science studies by framing $\alpha_{1}$ adrenergic blockers as a viable, potentially new treatment strategy in IPF.

\section{Discussion}

Because a major unmet need in the field of inflammatory remodeling is the discovery of intervenable processes driving fibrotic pathologies (1), our study aimed to uncover potentially new disease mechanisms that can be easily treated. The central discovery reported herein is that macrophage-derived NTN1 drives the development of fibrosis through a mechanism involving remodeled adrenergic nerves, their secretory product noradrenaline, and $\alpha_{1}$ adrenoreceptors; and that this axis is at least partially intact in humans with IPF where it may be targeted with safe and well-tolerated $\alpha_{1}$ adrenoreceptor antagonists that are already FDA approved. Our work has relevance for scarring conditions affecting the lung, including IPF and the related and often fatal parenchymal disorders arising in the setting of connective tissue disease, sarcoidosis, toxins and irradiation, and acute interstitial pneumonia. Because macrophages and/or NGPs are implicated in the inflammatory remodeling of multiple organs, our work has significance for diseases affecting the liver, kidney, skin, and bone marrow as well. Finally, because adrenergic innervation has been linked to cancer and obesity, our work may be relevant in these domains.

We used the lung as a modeling system to introduce several potentially new concepts in the field of fibrosis. The first potentially new idea is that the development of fibrosis is driven by adrenergic nerves. Despite the increasingly recognized contribution of adrenergic innervation to pathologies of the bone marrow (43), adipose tissue (12), liver (44), and some malignancies (45), its role in fibrotic repair is undefined. Based upon the timing of noradrenaline accumulation, the accumulation of ADRA1expressing hematopoietic and stromal cells, and the response to both chemical denervation and delayed initiation of treatment in the mouse model, we propose that nerve-derived noradrenaline operates downstream of injury and inflammatory cell recruitment to impact the fibroproliferative aspects of disease. This could occur either through direct activation of the ADRA1 receptors, indirectly via noradrenaline uptake and recycling via solute carrier family 6 member 2 and monoamine oxidase A (12), or via additional mechanisms that have yet to be defined. The improved outcomes seen in IPF patients prescribed $\alpha_{1}$ adrenoreceptor antagonists suggest real clinical impact of our work. Given the widespread adrenergic innervation that exists in the lung and elsewhere, it is likely that additional processes affecting blood vessels, airways, and distal organs are implicated. Other forms of autonomic innervation are undoubtedly involved, as vagotomy in animal models has been shown to prevent fibrosis (46) and suppress NTN1 (25). How these observations are linked to the adrenergic system remains unknown. Nevertheless, given the robust suppression of fibrosis and improved outcomes achieved via therapeutic $\alpha_{1}$ blockade in mice and humans, respectively, it is easy to envision the relatively rapid repurposing of this drug class for fibrotic indications such as IPF.

The current study tested the novel hypothesis that NGPs participate in fibrosis via their axon-related functions and discovered that the prototypical NGP, NTN1, mediates fibrosis and stimulates adrenergic nerve remodeling. NGPs are implicated as drivers of apoptosis (47); proliferation and survival (47); production of 18-glycosyl hydrolase proteins (48); protection of mitochondrial integrity and function (49); recruitment, retention, and activation of immune cells (50-52); activation of TGF- $\beta 1$ (50); and production and secretion of lipid mediators (25). Our study adds to this body of literature by demonstrating a role for myeloid-derived NTN1 in nerve remodeling, neurotransmitter accumulation, and fibrosis. NTN1 could act on nerve sprouting, migration, and/or survival, or could function indirectly through effects on neighboring cells. The reduction of fibrosis in the $D c c^{\text {kanga/+ }}$ mice suggests the former function, although we have not evaluated a role for DCC in other cell populations or parts of the fibrotic response. Further study is required to determine the validity of this hypothesis and whether it extends to other NGPs.

We also report the provocative finding that macrophages contribute to postinjury pulmonary nerve remodeling. Recent work reports the presence of nerve-associated macrophages in multiple organs, including the lung $(10,12)$; however, ours is the first study, to our knowledge, to show that macrophages contribute to nerve homeostasis in the adult lungs in a manner reminiscent of microglial function in the central nervous system. We targeted Ntn1 in LysM-Cre ${ }^{+}$cells throughout development using the Lyz2 promoter. This promoter is primarily active in cells of myeloid lineage (28) and to a much smaller extent in alveolar epithelial type II cells. We used multiple complementary methods to identify the critical 

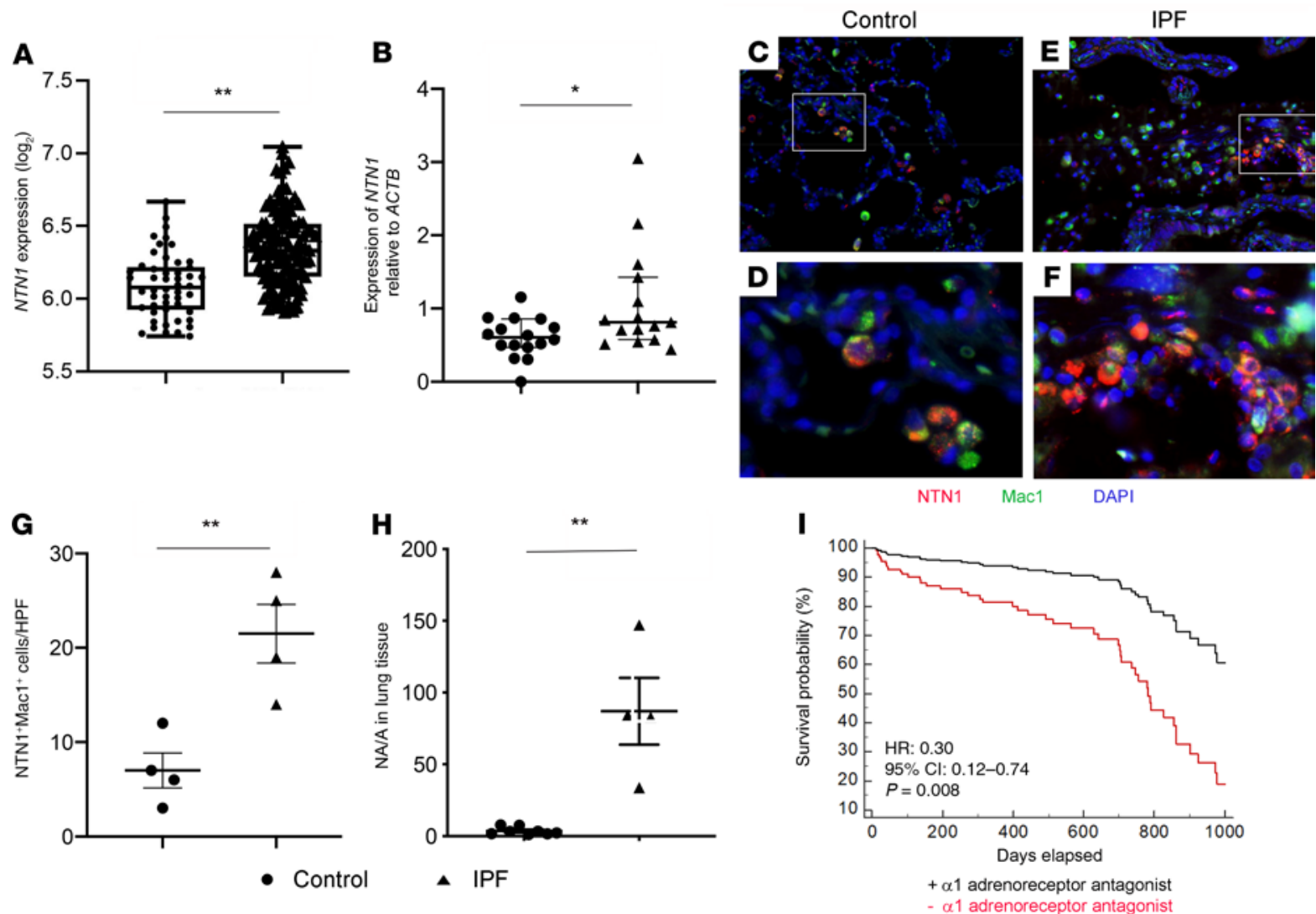

Figure 5. NTN1 expression, noradrenaline content, and effect of $\alpha_{1}$ adrenoreceptor antagonist use on survival in human IPF. (A) Evaluation of publicly available microarray data from the LGRC cohort revealed that relative to the controls ( $n=50$, round symbol), IPF lungs ( $n=119$, triangle) exhibited higher NTN1 expression ( $6.3 \pm 0.3$ vs. $6.1 \pm 0.2$, $\left[\log _{2}\right]$, FDR-adjusted $P<0.01$ ). (B) Compared with the control, the expression of NTN1 relative to ACTB was increased in IPF lung tissues ( $P=0.0398$, Mann-Whitney comparison). ( $\mathbf{C}-\mathbf{F}$ ) Immunofluorescence detection of NTN1 (red) and Mac1 (green) in archived lung biopsy specimens from controls ( $\mathbf{C}$ and $\mathbf{D})$ and IPF patients (E and $\mathbf{F})$. The boxed areas in the low-power views are shown directly beneath. Slides were counterstained with DAPI (blue). Original magnification, $\times 20$. (C) Compared with control samples, the number of Mac $1^{+}$cells coexpressing NTN1 counted per high-powered field (HPF) was increased in IPF ( $P=0.0073$, 2-tailed Student's $t$ test). (H) Compared with explanted donor lung tissues, the NA/A ratio was increased in IPF lungs obtained at the time of transplant $(P=0.003$, 2-tailed Student's $t$ test). (I) Kaplan-Meier analysis adjusted for age, sex, FVC, DLCO, and GAP index comparing patients prescribed an $\alpha_{1}$ adrenergic receptor antagonist (black line, $n=22$ ) to those not on such therapy (red line, $n=67$ ) showed a hazard ratio for all-cause mortality of $0.30(P=0.008) .{ }^{*} P<0.05,{ }^{* *} P<0.01$, and ${ }^{* *} P<0.001$. Data are shown as either median with $95 \%$ confidence interval or mean $\pm \mathrm{SEM}$.

$\mathrm{NTN1} 1^{+}$-expressing population as macrophages, but cannot rule out the contribution from other $L y z 2^{+}$cells. We also cannot rule out other functions of macrophages and cannot, with any certainty, make conjectures regarding the effector myeloid/macrophage population. However, the immunostaining results from both the mouse and human work would suggest at least a partial contribution of lung-resident, long-lived, yolk sac-derived alveolar macrophages (53). Further work will be required to determine the identity of these cells and how they can best be targeted in human disease, and whether NTN1 produced by additional populations such as fibroblasts, epithelia, and endothelia at different periods of fibrogenesis is similarly implicated in this process.

The limitations of our work constitute the framework for multiple new areas of related investigation. The potential benefit of $\alpha_{1}$ adrenergic antagonism will require replication in prospectively conducted, appropriately controlled studies including female patients. The effect of noradrenaline on ADRA1 receptor-expressing stromal and immune cells requires further investigation, and the role of other nerve-derived catecholamines such as dopamine should also be assessed (54). Unraveling the benefit of myeloid Ntn1 deletion will require the generation of bone marrow chimeras and use of inducible macrophagespecific promoters to avoid undetected developmental or off-target effects. The specificity of the NTN1/DCC interaction would be better studied with the use of inducible $D c c$ deletion in $\mathrm{TH}^{+}$ cells and evaluation of additional cell populations expressing DCC. The origin and identity of macrophages that can remodel adrenergic nerves have yet to be identified. Finally, the relevance of our observations to other fibrosing disorders will require study in additional disease models and patient cohorts.

In conclusion, we have described a process of adrenergic nerve-associated lung fibrosis that is driven by macrophagederived NTN1 and responds to treatment with $\alpha_{1}$ adrenoreceptor blockade. Because NTN1-expressing macrophages have been identified in numerous contexts, and the medications used in our study are available for nonfibrotic indications, our findings are of broad importance with high clinical relevance. A better understanding of the mechanism through which myeloid NTN1 regulates adrenergic nerve remodeling and downstream $\alpha_{1}$ adrenergic receptor activation holds promise for the treatment of fibrotic disease. 
Table 2. Demographic characteristics of IPF patients from TYLR

(-) $\alpha 1$ adrenergic antagonist

67 Age (years)

\author{
Mean \pm SD
}

Race/ethnicity $n$ (\%)

White

African American

Hispanic

Smoking status $\boldsymbol{n}(\%)$

Ever/Current

Never

FVC\% at enrollment

(Percentage predicted mean \pm SD)

DLCO $\% \mathrm{Hgb}$ at enrollment

(Percentage predicted mean \pm SD)

GAP index at enrollment

(Percentage predicted mean \pm SD)

$\alpha 1$ adrenergic antagonist $n(\%)$

Tamsulosin

Terazosin

$71.19 \pm 6.83$

$62(92.54)$

2 (2.98)

$3(4.48)$

$12(17.91)$

$4.64 \pm 1.33$
(+) $\alpha 1$ adrenergic antagonist

\section{$P$ value}

22

$74.16 \pm 5.29$

0.067

21 (95.45)

$1(4.55)$

$0(0.00)$

$18(81.82)$

$4(18.18)$

$83.00 \pm 16.83$

0.009

$41.88 \pm 15.19 \quad 51.18 \pm 17.69$

$3.96 \pm 0.99$

0.029

$17(77.2)$

$5(22.8)$

Data are presented as mean \pm SD. FVC\% = percentage of predicted forced vital capacity. DLCO\% = percentage of predicted diffusion capacity of the lung for carbon monoxide. GAP = gender, age, physiology.

\section{Methods}

Detailed methods are available in Supplemental Methods.

Study design. Power analysis was performed for the in vivo studies. All data were included in the final analysis, and outliers were not excluded. Primary endpoints were prospectively determined, and appropriate corrections were made for multiple testing. Mouse studies were repeated twice. Randomization occurred manually. The human studies were performed once. The objectives of our study were to determine the mechanism through which NTN1 drives pulmonary fibrosis and to test the prespecified hypothesis that its role involves adrenergic nerve remodeling. The units of investigation included genetically modified and WT mice, existing data sets, and archived samples and data from healthy control and IPF participants. The overall design involved evaluation of fibrosis in genetically modified mice or in WT animals subjected to chemical denervation or $\alpha_{1}$ blocker treatment. Archived samples and historical data from humans were used to support the clinical relevance of our experimental observations. Analysis was performed in triplicate using randomization to avoid block effect. Mice and samples were numerically coded, and animal handlers and laboratory staff were blinded to the study group.

Animals. Mice were backcrossed for more than 10 generations onto a C57BL/6J background. Male and female mice were used between age 7 and 9 weeks. WT, ColIa2-CreER (55), LysM-Cre (28), and Dcc kanga/+ (33) mice were purchased from The Jackson Laboratory. $N \operatorname{tn} 1^{f / f l}(56)$ and $\mathrm{Ntn1}^{+/-}$(16) mice were described previously.

Drugs. Pharmacological grade bleomycin was obtained from McKesson Medical Surgical, Inc. 6-OHDA (H4381), terazosin (T4680), tamoxifen (T5648), and urethane (U2500) were purchased from MilliporeSigma. Isoflurane (50019100) was ordered from Zoetis Inc.; 1,2-propanediol (30948) was purchased from Alfa Aesar.

Bleomycin models. Bleomycin administration, BAL, and lung harvest were performed according to our previous description (57). Unless stated otherwise, groups were evenly divided between males and females.
Collagen quantification. Collagen content was assessed by Sircol Soluble Collagen Assay (Biocolor Ltd., CLS 1111) according to our previous methods (31).

Vibratome sectioning and staining. Vibratome sectioning and immunofluorescence staining were carried out using either the pan-neuronal marker PGP9.5 or for the rate-limiting enzyme in the biosynthesis of catecholamines, $\mathrm{TH}$, as a sympathetic neuron marker. Lung preparation for vibratome sectioning was performed as previously described (58). The 150- $\mu \mathrm{m}$-thick lung sections were blocked overnight with $5 \%$ normal goat serum (NGS) in $0.5 \%$ Triton X-100/1× PBS (PBS-T) at $4^{\circ} \mathrm{C}$ on a shaker. Sections were then incubated with rabbit anti-TH (1:100, Abcam, ab112) and mouse anti- $\alpha$-SMA (1:250, Abcam, ab7817) antibodies for 3 days at $4^{\circ} \mathrm{C}$. The colocalization staining was carried out using rabbit anti-TH and mouse anti-PGP9.5 (1:100, Abcam, ab8189). After the washing with PBS-T, lung sections were continuously incubated overnight with the corresponding Alexa Fluor-488 goat anti-rabbit IgG (1:500, Invitrogen, A11008) and Alexa Fluor 555-goat anti-mouse IgG (1:500, Invitrogen, A21422) for 24 hours at $4^{\circ} \mathrm{C}$. Finally, lung sections were washed 4 times with PBS-T and mounted with the VECTASHIELD antifade mounting medium containing $1.5 \mu \mathrm{g} / \mathrm{mL}$ DAPI (Vector Laboratories, H-1200). Images were taken using an SP8 confocal laser microscope with the software of Leica Application Suite X (Leica Microsystems Inc.), and TH fibers were quantified visually in serial sections of multiple lobes as fibers/ $\mathrm{mm}^{3} /$ tissue. Antibodies are listed in Supplemental Table 2.

Imaging mass cytometry. After dewaxing and rehydration, tissue sections were blocked with $0.3 \%$ BSA in Dulbecco's PBS (DPBS) for 30 minutes at room temperature in a hydration chamber and then incubated overnight with the prepared antibody cocktail (Supplemental Table 3, Netrin-1-152Sm, CD11b-149Sm, F4/80-159Tb, and Histone $\mathrm{H} 3-176 \mathrm{Yb}$ [to identify nuclei]) in $100 \mu \mathrm{L}$ DPBS at $4^{\circ} \mathrm{C}$ in a hydration chamber. The next day, slides were washed, and sections 
were stained with Ir-Intercalator in DPBS for 60 minutes at room temperature in a hydration chamber, then washed with TBS-T for 5 minutes (twice), then washed with Milli-Qwater for 5 minutes (twice), and then allowed to air-dry for more than 30 minutes at room temperature. Slides were imaged with the Hyperion Imaging Mass Cytometer (27). Data were analyzed using Histology Topography Cytometry Analysis Toolbox (HistoCAT) (59). The fold change in cell density per $\mathrm{mm}^{2}$ was calculated and compared between groups.

Determination of noradrenaline and adrenaline concentrations in $B A L$ and lung tissues. Concentrations of noradrenaline and adrenaline were determined in BAL and lung tissues using commercially available noradrenaline and adrenaline high-sensitivity ELISA kits (DLD Diagnostika, GMBH, NOU39-K01 and ADU39-K01) that have been used for this application in other publications (60).

Chemical sympathectomy. Chemical sympathectomy was performed to abrogate presynaptic noradrenaline release according to previous descriptions using 6-OHDA as the specific sympathetic neurotoxin (61).

FACS. FACS was performed on an LSRII flow cytometer on digested lung tissue suspensions prepared according to a method already in use in our lab (51). The mice of interest were euthanized and the lungs were flushed with $1 \times$ PBS as described above. Lungs were harvested, chopped into small pieces, and then digested in DMEM with high glucose containing $150 \mu \mathrm{g} / \mathrm{mL}$ collagenase (MilliporeSigma, C5138) and $20 \mathrm{U} / \mathrm{mL}$ DNase I (Roche, 04716728) for 1 hour at room temperature. This digestion was halted by addition of $1 \times$ PBS. The digested tissue was mechanically dissociated, filtered through a $100-\mu \mathrm{m}$ cell strainer, and then centrifuged at $495 \mathrm{~g}$ for 10 minutes at $4^{\circ} \mathrm{C}$. The supernatants were dumped, and $10 \mathrm{~mL}$ of $1 \times$ PBS were added into the pellets for cell counting. After centrifuging again, the pellets were resuspended in FACS buffer ( $1 \times$ PBS containing $2 \% \mathrm{FBS}, 0.01 \% \mathrm{NaN}_{3}$, and $1 \mathrm{mM}$ EDTA) at a density of $2 \times 10^{7}$ cells $/ \mathrm{mL}$. Next, $2 \times 10^{6}$ cells were taken and incubated with the surface antibodies in FACS buffer containing $10 \%$ NGS for 1 hour at $4^{\circ} \mathrm{C}$. Antibodies are shown in Supplemental Table 4. The following surface antibodies were used: rat CD45 mAb FITC (1:100, eBioscience, 11-0451-82), rat PE CD45 (1:100, BD Biosciences, 553081), rat CD45 mAb PerCP (1:1000, eBioscience, 45-0451-82), rat CD45 APC (1:100, eBioscience, 17-0451-82), rabbit anti-ADRA1A (1:5000, Abcam, ab22519), rabbit anti-ADRA1B (1:1000, Abcam, ab169523), rabbit alpha-1D adrenoceptor (1:100, Invitrogen, PA5-77286), rat PE CD31 (1:100, BD Biosciences, 553373), rat CD326 (EpCAM) (G8.8) APC (1:200, Invitrogen, 17-5791-82), rat PE anti-CD11b (1:200, BD Biosciences, 557397), Armenian-hamster CD11c (N418) PerCP-cyanine 5.5 (1:800, eBioscience, 45-0114-80), and rat F4/80 (BM8) APC (1.5:100, Invitrogen, 17-4801-82). After washing with FACS buffer, cell pellets were permeabilized in $500 \mu \mathrm{L}$ of $1 \times$ fixation/permeabilization buffer (eBioscience, 005123-43) overnight at $4^{\circ} \mathrm{C}$. The next day, cells were washed with $1 \times$ permeabilization buffer (eBioscience, 00-8333-56) and then continuously labeled with intracellular antibodies for 1 hour at room temperature. The following intracellular antibodies were used: rat FITC CD206 (MRC1) (1.5:100, BioLegend, 141704), goat anti-type I collagen (1:100, Southern Biotech, 1310-01), and mouse netrin-1-FITC (1:200, Novus Biologicals, NB600-1344F). Where relevant, secondary detection of unconjugated primary antibodies was then performed. Finally, cells were washed with FACS buffer and filtered through a $100-\mu \mathrm{m}$ cell strainer. Acquisition was performed on an LSRII flow cytometer using FACSDiva acquisition software. FlowJo software (FlowJo, LLC) was used for data analysis. The positive and negative gates were set based on relevant isotype control. Antibodies are listed in Supplemental Table 3.

Terazosin treatment. Briefly, mice received a single dose of vehicle or bleomycin $(1.25 \mathrm{U} / \mathrm{kg})$ on day 0 . Five days later, these mice were randomly divided into 2 groups. One group was i.p. injected with 1 $\mathrm{mg} / \mathrm{kg}$ terazosin from day 5 to day 13; another group was synchronously treated with saline and served as vehicle controls. Mice were euthanized on day 14. In later experiments, bleomycin-exposed mice were treated with terazosin or vehicle from day 10 to day 21 .

Histologic analysis, immunohistochemistry, and immunofluorescence. Whole left lungs were harvested from experimental mice for histological analysis. FFPE sections were stained with H\&E to assess gross morphology or Masson's trichrome stains to visualize collagen deposition (50). MAS was performed with a minor modification of previously published method (62). This quantitative scoring system assigns a numerical value between 0 and 8 that correspond to the extent of fibrosis in a histologic sample. NTN1 immunohistostaining was performed on unstained paraffin sections. NTN1 was detected with primary antibody (R\&D Systems, ab301324) followed by anti-rabbit HRP secondary antibody (Abcam, ab205718) and detected with a brown liquid $\mathrm{DAB}^{+}$ substrate chromogen system (Dako, 34688). Cell nuclei were counterstained with modified Meyer's hematoxylin (Abcam, ab220365). Immunofluorescence detection of NTN1 on human lung biopsy specimens was performed using goat polyclonal netrin-1 antibody (Novus, NBP1-51922) and rat monoclonal Mac1 antibody (Abcam, ab8878) followed by secondary detection and DAPI counterstaining.

RNA extraction, $c D N A$ synthesis, and quantitative real-time PCR. RNA isolation, cDNA reverse transcription, quantitative real-time $\mathrm{PCR}$, and relative quantification using the $2^{-\mathrm{\Delta CT}}$ method were carried out as previously reported by our lab (31).

TUNEL staining. Paraffin-embedded $5-\mu \mathrm{m}$ sections from FFPE lung tissues were detected by the TUNEL assay using the ApopTag Peroxidase in Situ Apoptosis Detection Kit (MilliporeSigma, S7100) according to the manufacturer's protocol, as we have reported (31).

The LGRC cohort. Gene expression data for the NTN1 gene were measured at the probe-level (probe ID: 8004880), from the Affymetrix Human Gene 1.0 ST Array (Affymetrix), and used in the LGRC cohort as previously described (40).

Data and materials availability. All materials reported in this manuscript are either commercially available or can be obtained under a materials transfer agreement. Apart from FACS data showing absent ADRA1 receptor expression on epithelium and endothelium, all the data are available in the main text and Supplemental Methods.

Statistics. Parametric data are graphed as mean \pm SEM and were analyzed using GraphPad Prism version 7.01. Nonparametric data are graphed as median with IQR. Multiple comparisons were achieved using 2-way ANOVA with appropriate multiple-comparisons test. Comparisons between 2 groups were made using an unpaired 2-tailed Mann-Whitney comparison or Student's $t$ test. For LGRC and TYLR data, R software (R Foundation for Statistical Computing) was used for data analysis. The R stats package version 3.4.3 was implemented. Results were reported as medians and IQRs (25\% to $75 \%$ ) unless otherwise specified. For the LGRC cohort, the $P$ value for NTN1 represents probe-level statistics adjusted for multiple comparisons. Continuous variables were tested using nonparametric tests, including the Kruskal-Wallis test. Categorical variables were analyzed with Fisher's exact test. Kaplan-Meier analysis determined 
survival associations. Multivariate Cox regression hazards ratio was used to model association with relevant covariates. $P$ values of less than 0.05 after multiple-comparisons testing (where relevant) were considered statistically significant.

Study approval. These studies were performed with approval from the Yale IACUC and IRB. All animal experiments were performed according to the principles approved by the Yale University IACUC with handling adhering to the Guide for the Care and Use of Laboratory Animals. All human studies were performed with approval from institutional human investigation committee(s) at Yale University or Massachusetts General Hospital, and written informed consent was received from participants before inclusion in the study.

\section{Author contributions}

RG conducted the investigation, methodology, formal analysis, and visualization and reviewed and edited the manuscript. XP, $\mathrm{CP}, \mathrm{HS}, \mathrm{AN}, \mathrm{BR}, \mathrm{AW}$, and MWM performed the investigation. CR performed the investigation and formal analysis. NK, NN, GI, and JLG conducted formal analysis. KB and LH provided resources. MG conducted the investigation and clinical phenotyping. RJH performed the investigation and reviewed and edited the manuscript. DMG and HKE provided resources and reviewed and edited the manuscript. ELH provided conceptualization, resources, visualization, supervision, project administration, and funding acquisition and wrote the manuscript.

\section{Acknowledgments}

We thank Anne Eichmann for intellectual support of the project, provision of mice and reagents, and critical reading of the manuscript. These studies were funded by the following: the Parker B. Francis Foundation, the Foundation for Sarcoidosis Research, and 1K08HL151970 (to CR); ATS Foundation: AWD0002487, Scleroderma Foundation, and DOD PR192454 (to HS); R01 HL125815, HL133016, HL142674, and DOD W81XWH1810629 (to DMG); R01 DK097075, HL114457, HL109233, DK109574, HL119837, and HL133900 (to HKE); R01 HL109233, HL125850, HL152677, DOD PR170078, the Gabriel and Alma Elias Research Fund, and the Greenfield Foundation (to ELH).

Address correspondence to: Erica L. Herzog, Yale School of Medicine, 300 Cedar Street TAC 441S, New Haven, Connecticut 065208057, USA. Phone: 203.785.3627; Email: erica.herzog@yale.edu.
1. Blackwell TS, et al. Future directions in idiopathic pulmonary fibrosis research. An NHLBI workshop report. Am J Respir Crit Care Med. 2014;189(2):214-222.

2. Rosenbloom J, Macarak E, Piera-Velazquez S, Jimenez SA. Human fibrotic diseases: current challenges in fibrosis research. Methods Mol Biol. 2017;1627:1-23.

3. Desai O, Winkler J, Minasyan M, Herzog EL. The role of immune and inflammatory cells in idiopathic pulmonary fibrosis. Front Med (Lausanne). 2018;5:43.

4. Murray LA, et al. TGF-beta driven lung fibrosis is macrophage dependent and blocked by serum amyloid P. Int JBiochem Cell Biol. 2011;43(1):154-162.

5 . Shook BA, et al. Myofibroblast proliferation and heterogeneity are supported by macrophages during skin repair. Science. 2018;362(6417):eaar2971.

6. Duffield JS, et al. Selective depletion of macrophages reveals distinct, opposing roles during liver injury and repair. J Clin Invest. 2005;115(1):56-65.

7. Lin SL, et al. Macrophage Wnt7b is critical for kidney repair and regeneration. Proc Natl Acad Sci U S A. 2010;107(9):4194-4199.

8. Schafer DP, Stevens B. Microglia function in central nervous system development and plasticity. Cold Spring Harb Perspect Biol. 2015;7(10):a020545.

9. Scheib J, Höke A. Advances in peripheral nerve regeneration. Nat Rev Neurol. 2013;9(12):668-676.

10. Chakarov S, et al. Two distinct interstitial macrophage populations coexist across tissues in specific subtissular niches. Science. 2019;363(6432):eaau0964.

11. Kolter J, Kierdorf K, Henneke P. Origin and differentiation of nerve-associated macrophages. J Immunol. 2020;204(2):271-279.

12. Camell CD, et al. Inflammasome-driven catecholamine catabolism in macrophages blunts lipolysis during ageing. Nature.
2017;550(7674):119-123.

13. Liu T, Yang L, Han X, Ding X, Li J, Yang J. Local sympathetic innervations modulate the lung innate immune responses. Sci Adv. 2020;6(20):eaay1497.

14. Ural BB, et al. Identification of a nerve-associated, lung-resident interstitial macrophage subset with distinct localization and immunoregulatory properties. Sci Immunol. 2020;5(45):eaax8756.

15. Mirakaj V, Rosenberger P. Immunomodulatory functions of neuronal guidance proteins. Trends Immunol. 2017;38(6):444-456.

16. Sun H, et al. Netrin-1 regulates fibrocyte accumulation in the decellularized fibrotic sclerodermatous lung microenvironment and in bleomycin-induced pulmonary fibrosis. Arthritis Rheumatol. 2016;68(5):1251-1261.

17. De Minicis S, et al. Semaphorin 7A contributes to TGF- $\beta$-mediated liver fibrogenesis. Am J Pathol. 2013;183(3):820-830.

18. Ramkhelawon B, et al. Netrin-1 promotes adipose tissue macrophage retention and insulin resistance in obesity. Nat Med. 2014;20(4):377-384

19. Lee Y, et al. Semaphorin 7A in circulating regulatory $\mathrm{T}$ cells is increased in autosomal-dominant polycystic kidney disease and decreases with tolvaptan treatment. Clin Exp Nephrol. 2018;22(4):906-916.

20. Serafini T, Kennedy TE, Galko MJ, Mirzayan C, Jessell TM, Tessier-Lavigne M. The netrins define a family of axon outgrowth-promoting proteins homologous to C. elegans UNC-6. Cell. 1994;78(3):409-424.

21. Kolodkin AL, Matthes DJ, Goodman CS. The semaphorin genes encode a family of transmembrane and secreted growth cone guidance molecules. Cell. 1993;75(7):1389-1399.

22. Jaminet $P$, et al. Evaluating the role of Netrin-1 during the early phase of peripheral nerve regeneration using the mouse median nerve model. Restor Neurol Neurosci. 2013;31(3):337-345.
23. Ke X, et al. Netrin-1 overexpression in bone marrow mesenchymal stem cells promotes functional recovery in a rat model of peripheral nerve injury. J Biomed Res. 2015;29(5):380-389.

24 . Brunet I, et al. Netrin-1 controls sympathetic arterial innervation. JClin Invest. 2014;124(7):3230-3240.

25. Mirakaj V, Dalli J, Granja T, Rosenberger P, Serhan CN. Vagus nerve controls resolution and pro-resolving mediators of inflammation. J Exp Med. 2014;211(6):1037-1048.

26. Borovikova LV, et al. Vagus nerve stimulation attenuates the systemic inflammatory response to endotoxin. Nature. 2000;405(6785):458-462.

27. Giesen C, et al. Highly multiplexed imaging of tumor tissues with subcellular resolution by mass cytometry. Nat Methods. 2014;11(4):417-422.

28. Clausen BE, Burkhardt C, Reith W, Renkawitz $\mathrm{R}$, Förster I. Conditional gene targeting in macrophages and granulocytes using LysMcre mice. Transgenic Res. 1999;8(4):265-277.

29. Ranganathan P, Jayakumar C, Santhakumar M, Ramesh G. Netrin-1 regulates colon-kidney cross talk through suppression of IL-6 function in a mouse model of DSS-colitis. Am J Physiol Renal Physiol. 2013;304(9):F1187-F1197.

30. Aherne CM, et al. Neuronal guidance molecule netrin-1 attenuates inflammatory cell trafficking during acute experimental colitis. Gut. 2012;61(5):695-705.

31. Zhou Y, et al. Chitinase 3-like 1 suppresses injury and promotes fibroproliferative responses in mammalian lung fibrosis. Sci Transl Med. 2014;6(240):240ra76.

32. Tieu K. A guide to neurotoxic animal models of Parkinson's disease. Cold Spring Harb Perspect Med. 2011;1(1):a009316.

33. Flores C, et al. Netrin receptor deficient mice exhibit functional reorganization of dopaminergic systems and do not sensitize to amphetamine. Mol Psychiatry. 2005;10(6):606-612.

34. Piascik MT, Perez DM. Alpha1-adrenergic recep- 
tors: new insights and directions. J Pharmacol Exp Ther. 2001;298(2):403-410.

35. Rassler B, Marx G, Schierle K, Zimmer HG. Catecholamines can induce pulmonary remodeling in rats. Cell Physiol Biochem. 2012;30(5):1134-1147.

36. Ribeiro-da-Silva $M$, et al. Interplay between sympathetic nervous system and inflammation in aseptic loosening of hip joint replacement. Sci Rep. 2018;8(1):16044.

37. Stofkova A, Kamimura D, Ohki T, Ota M, Arima Y, Murakami M. Photopic light-mediated down-regulation of local $\alpha_{1 \mathrm{~A}}$-adrenergic signaling protects blood-retina barrier in experimental autoimmune uveoretinitis. Sci Rep. 2019;9(1):2353.

38. Muramatsu I, Taniguchi T, Okada K. Tamsulosin: alpha1-adrenoceptor subtype-selectivity and comparison with terazosin. Jpn JPharmacol. 1998;78(3):331-335.

39. Maher TM, Strek ME. Antifibrotic therapy for idiopathic pulmonary fibrosis: time to treat. Respir Res. 2019;20(1):205.

40. Yang IV, et al. Expression of cilium-associated genes defines novel molecular subtypes of idiopathic pulmonary fibrosis. Thorax. 2013;68(12):1114-1121.

41. Raghu G, et al. An official ATS/ERS/JRS/ ALAT statement: idiopathic pulmonary fibrosis: evidence-based guidelines for diagnosis and management. Am J Respir Crit Care Med. 2011;183(6):788-824.

42. Ley B, et al. A multidimensional index and staging system for idiopathic pulmonary fibrosis. Ann Intern Med. 2012;156(10):684-691.

43. Maryanovich $M$, et al. Adrenergic nerve degeneration in bone marrow drives aging of the hematopoietic stem cell niche. Nat Med. 2018;24(6):782-791.
44. Lin JC, Peng YJ, Wang SY, Young TH, Salter DM, Lee HS. Role of the sympathetic nervous system in carbon tetrachloride-induced hepatotoxicity and systemic inflammation. PLoS One. 2015;10(3):e0121365.

45. Zahalka AH, et al. Adrenergic nerves activate an angio-metabolic switch in prostate cancer. Science. 2017;358(6361):321-326.

46. Song N, et al. Vagotomy attenuates bleomycin-induced pulmonary fibrosis in mice. Sci Rep. 2015;5:13419.

47. Kang HR, Lee CG, Homer RJ, Elias JA. Semaphorin 7A plays a critical role in TGF-beta1induced pulmonary fibrosis. J Exp Med. 2007;204(5):1083-1093.

48. Ma B, et al. Role of chitinase 3-like-1 and semaphorin 7 a in pulmonary melanoma metastasis. Cancer Res. 2015;75(3):487-496.

49. Bouhidel JO, Wang P, Siu KL, Li H, Youn JY, Cai H. Netrin-1 improves post-injury cardiac function in vivo via DCC/NO-dependent preservation of mitochondrial integrity, while attenuating autophagy. Biochim Biophys Acta. 2015;1852(2):277-289.

50. Gan Y, et al. Role of semaphorin 7a signaling in transforming growth factor $\beta 1$-induced lung fibrosis and scleroderma-related interstitial lung disease. Arthritis Rheum. 2011;63(8):2484-2494.

51. Peng X, et al. Plexin C1 deficiency permits synaptotagmin 7-mediated macrophage migration and enhances mammalian lung fibrosis. FASEB J. 2016;30(12):4056-4070.

52. Reilkoff RA, et al. Semaphorin $7 \mathrm{a}^{+}$regulatory $\mathrm{T}$ cells are associated with progressive idiopathic pulmonary fibrosis and are implicated in transforming growth factor- $\beta 1$-induced pulmonary fibrosis. Am J Respir Crit Care Med. 2013;187(2):180-188.
53. Tan SY, Krasnow MA. Developmental origin of lung macrophage diversity. Development. 2016;143(8):1318-1327.

54. Haak AJ, et al. Selective YAP/TAZ inhibition in fibroblasts via dopamine receptor D1 agonism reverses fibrosis. Sci Transl Med. 2019;11(516):eaau6296.

55. Xie T, et al. Transcription factor TBX4 regulates myofibroblast accumulation and lung fibrosis. JClin Invest. 2016;126(8):3063-3079.

56 . Hadi T, et al. Macrophage-derived netrin-1 promotes abdominal aortic aneurysm formation by activating MMP3 in vascular smooth muscle cells. Nat Commun. 2018;9(1):5022.

57. Peng $X$, et al. Plexin C1 deficiency permits synaptotagmin 7-mediated macrophage migration and enhances mammalian lung fibrosis. FASEB $J$. 2016;30(12):4056-4070.

58. Sheikh AQ, Saddouk FZ, Ntokou A, Mazurek $\mathrm{R}$, Greif DM. Cell autonomous and non-cell autonomous regulation of SMC progenitors in pulmonary hypertension. Cell Rep. 2018;23(4):1152-1165.

59. Schapiro D, et al. histoCAT: analysis of cell phenotypes and interactions in multiplex image cytometry data. Nat Methods. 2017;14(9):873-876.

60. Nguyen KD, et al. Alternatively activated macrophages produce catecholamines to sustain adaptive thermogenesis. Nature. 2011;480(7375):104-108.

61. Grebe KM, et al. Cutting edge: Sympathetic nervous system increases proinflammatory cytokines and exacerbates influenza A virus pathogenesis. JImmunol. 2010;184(2):540-544.

62. Hübner RH, et al. Standardized quantification of pulmonary fibrosis in histological samples. BioTechniques. 2008;44(4):507-511, 514. 
